\title{
Conocimientos sobre tuberculosis en estudiantes de enfermeria de una universidad colombiana
}

\author{
Knowledge of tuberculosis in nursing \\ students' university of Colombia
}

\author{
Gladia Lorena Ortega Barón¹, Paola Andrea Rodríguez Quezadaํㅗ Elver Camilo Jiménez Beltrán¹, \\ Alba Idaly Muñoz Sánchez ${ }^{1}$
}

\begin{abstract}
Forma de citar: Ortega Barón GL, Rodríguez Quesada PA, Jiménez Beltrán EC, Muñoz Sánchez AI. Conocimientos sobre tuberculosis en estudiantes de enfermería de una universidad colombiana. Rev Univ Ind Santander Salud. 2015; 47(3): 261-270. DOI: http://dx.doi.org/10.18273/revsal.v47n3-2015002 @c) (1) $\Theta$
\end{abstract}

\section{RESUMEN}

Objetivo: Describir los conocimientos sobre Tuberculosis (TB) en estudiantes de enfermería de una universidad de Bogotá, Colombia, período 2013-II. Materiales y Métodos: Estudio descriptivo transversal. Se elaboró una herramienta para la recolección de la información, constituida por 35 preguntas (18 sociodemográficas y 17 de conocimientos). Los datos se procesaron en el programa estadístico SPSS Versión 21, en el que se analizaron los conocimientos utilizando el coeficiente de correlación de Spearman para determinar las relaciones. Se preservaron los aspectos éticos de la investigación de acuerdo a las directrices establecidas por la Facultad de Enfermería de la Universidad Nacional de Colombia. Resultados: Participaron 119 estudiantes (43 de las primeras tres matrículas y 76 de la octava en adelante). Los estudiantes de las últimas matrículas tuvieron más aciertos en sus respuestas, comparado con los estudiantes de las primeras tres. Utilizando el coeficiente de correlación de Spearman y relacionando las matrículas con los conocimientos, se obtuvo un rho de 0,68. Conclusiones: Se encontraron falencias en los conocimientos sobre la TB de los estudiantes de enfermería participantes en temas como la utilidad de la vacuna BCG (Bacillus Calmette-Guérin), la sintomatología, la estrategia Directly Observed Treatment Short-course/Tratamiento Acortado Estrictamente Supervisado (DOTS/TAES), el tratamiento y las farmacorresistencias. Se considera la necesidad de orientar esfuerzos desde la formación para fortalecer los conocimientos.

Palabras clave: Investigación en Educación de Enfermería; Conocimientos, Actitudes y Prácticas en Salud; Estudiantes de Enfermería; Tuberculosis.

1. Universidad Nacional de Colombia, Bogotá.

Correspondencia: Alba Idaly Muñoz Sánchez. Dirección: Carrera 30 No. 45-03, edificio 101. Correo electrónico: albaidalymunoz@gmail.com. Teléfono: +1 3165000 ext. 17082 


\section{ABSTRACT}

Objective: To identify the knowledge of Tuberculosis (TB) in nursing students from an university in Bogotá, Colombia, in period 2013-II. Materials and Method: Quantitative, descriptive and transversal study. Information was collected based on a previously developed questionnaire, consisting on 35 questions (18 of them sociodemographic and 17 testing the knowledge). Data was then processed in the statistical program SPSS Version 21, knowledge was analyzed using Spearman correlation coefficient to determine relationships. Ethical aspects of the research were preserved. Results: 119 students participated (43 of them belonging to the first three enrollments and 76 of them to the eighth enrollment onwards). Students belonging to the lasts enrollments had a higher number of hits in their responses, compared with students of the first three. There were significant differences between the groups. Using Spearman correlation coefficient and relating the enrollments with the knowledge a rho of 0,68 was obtained. Conclusions: It was found shortcomings in the knowledge related to TB between participating nursing students on issues such as the usefulness of BCG vaccine (Bacillus Calmette-Guérin), symptoms, the Directly Observed Treatment Short-Course Strategy (DOTS), treatment and drug resistance. It was considered the need to focus efforts to improve training and strengthen the knowledge.

Keywords: Tuberculosis; Nursing Education Research; Health Knowledge, Attitudes, Practice; Students, Nursing.

\section{INTRODUCCIÓN}

La tuberculosis (TB) es clasificada por la Organización Mundial de la Salud (OMS) como una enfermedad infecciosa de importancia en salud pública, pues se estima que un tercio de la población mundial se encuentra infectada por el Mycobacterium tuberculosis y un $10 \%$ desarrollará la enfermedad en el futuro. Para el año 2013 se reportaron más de 9 millones de casos nuevos y 1,5 millones de muertes, de las cuales 360.000 correspondían a la coinfección TB-VIH, siendo la TB la segunda enfermedad infectocontagiosa que más muertes produce al año, después del VIH/SIDA (Virus de Inmunodeficiencia Adquirida/Síndrome de Inmunodeficiencia Adquirida) ${ }^{1}$.

En las Américas, aunque haya sido una de las primeras regiones en cumplir con los Objetivos de Desarrollo del Milenio (ODM) al reducir la carga de TB, durante el 2012 se reportaron alrededor de 208845 enfermos, con una tasa de incidencia de 28 casos por cada 100 000 habitantes $^{1}$. Aproximadamente $67 \%$ de los casos nuevos ocurrieron en América del Sur, cuyos mayores índices $(29 \%)$ se presentaron en la subregión andina -Colombia, Venezuela, Perú, Ecuador y Bolivia- ${ }^{2}$. En Colombia en el 2013 se reportaron 13486 casos según el Sistema Nacional de Vigilancia en Salud Pública (SIVIGILA), a partir de lo cual se destaca una reducción de las cifras en los últimos años, aunque no se ha logrado el control de la enfermedad ${ }^{3}$. A pesar de los esfuerzos de los diferentes organismos en salud y las metas fijadas en los ODM por la ONU para el 2015, la TB actualmente es la segunda causa de muerte por enfermedad infectocontagiosa después del VIH en el mundo ${ }^{1}$.
Por otra parte, en la formación del estudiante de enfermería se hace necesario el aprendizaje sólido de los conocimientos y la constante actualización para afrontar la TB y, de acuerdo con éstos, realizar las prácticas apropiadas para combatir la carga por esta enfermedad, disminuyendo la incidencia, la prevalencia, la aparición de cepas farmacorresistentes y la mortalidad ${ }^{4}$. Ahora bien, durante el proceso de formación, los estudiantes de enfermería se encuentran expuestos al agente infeccioso Mycobacterium tuberculosis al brindar el cuidado a los pacientes enfermos, por lo que los riesgos de infección aumentan al carecer de los conocimientos suficientes sobre la enfermedad, las medidas de protección y las políticas laborales adecuadas en los establecimientos de salud 5 .

Dentro de los estudios CAP (Conocimientos, Actitudes y Prácticas) en salud se identifican los conocimientos en una población determinada y, en este caso, cómo éstos revelan conductas individuales y colectivas en razón de las prácticas en TB. Un estudio de esta categoría se basa en una serie de preguntas abiertas o cerradas, en el que se recopila la información a través de entrevistas o cuestionarios previamente construidos. Estudios previos de conocimientos en estudiantes y profesionales del área de la salud en países como Brasil, Estados Unidos, India, Italia, Turquía, Irán, China, Sudáfrica, Cuba y Colombia han demostrado que existen bajos niveles de conocimientos en temáticas sobre $\mathrm{TB}$, como utilidad de la vacuna BCG, agente causal, transmisión, definición clínica del sintomático respiratorio, diagnóstico de la enfermedad, estrategia DOTS/TAES, régimen de tratamiento, programas de control de la TB en sus respectivos países, manejo de efectos secundarios y duración y control de la TB farmacorresistente ${ }^{7-11}$. $\mathrm{Al}$ identificar que Colombia carece de estudios CAP en 
estudiantes de enfermería, se realizó esta investigación con el objetivo de describir los conocimientos sobre TB. Teniendo en cuenta la experiencia práctica, se estudiaron dos grupos: el primero se compuso de estudiantes de las primeras tres matrículas y el segundo, de la octava en adelante, para lo cual se observó si éstos mejoraban a medida que la formación avanza. Al ser la presente investigación en la temática una de las primeras en el país, puede fomentar a futuro estudios de intervención como el desarrollo de estrategias educativas.

\section{MATERIALES Y MÉTODOS}

Estudio descriptivo transversal, en el cual se identificaron los conocimientos sobre TB en estudiantes de pregrado de enfermería, de acuerdo al número de matrículas cursadas, en una universidad de Bogotá, Colombia. La información se recolectó entre octubre del 2013 y enero del 2014.

\section{Herramienta para la recolección de la información}

Se elaboró una herramienta para la recolección de los datos diseñada por el equipo de investigadores, la cual se compuso de 17 preguntas de selección múltiple con única respuesta para la variable conocimientos en TB y 18 preguntas abiertas y de selección múltiple para la variable. Aspectos sociodemográficos. Para esto, se incluyeron preguntas con respecto a matrícula, edad, género, estado civil, procedencia, hijos, si laboraba en el momento de la investigación y si durante la formación académica había recibido información acerca de la tuberculosis. En conocimientos se abordaron preguntas sobre la TB respectivas al agente etiológico, la vacuna BCG, las vías de transmisión, la estrategia DOTS/ TAES, las farmacorresistencias, el tratamiento, la duración para casos nuevos y la definición clínica de sintomático respiratorio.

Para la formulación de las preguntas se tuvieron en cuenta los estudios CAP previos $^{5-10}$, la Guía para la elaboración de CAP de la $\mathrm{OMS}^{6}$, el Informe Mundial de TB OMS 2013르 , el Plan Estratégico de Colombia Libre de TB 2010-2015 ${ }^{13}$ y la Resolución 412 de 2000 del Ministerio de Salud, Colombia ${ }^{14}$.

El proceso de recolección de los datos se fundamentó en la información suministrada por la facultad seleccionada, en términos de estudiantes matriculados, y a los cuales se obtuvo acceso en horarios en los que se desarrollaban las actividades académicas para garantizar una amplia participación sin selección aleatoria de los sujetos. Como estrategia, se recopiló la información en dos momentos:

- Horas de clase: se solicitó la autorización de los docentes para informar acerca del proceso y la aplicación de la herramienta. Cada estudiante que cumpliera con los criterios de inclusión y aprobara su participación, resolvía cada una de las preguntas.

- Horas libres: por practicidad, se contactó individualmente a los estudiantes de la facultad en espacios no académicos, aplicando el mismo proceso de recolección de datos.

Los estudiantes diligenciaron la herramienta de manera individual y voluntaria, sin el acceso a recursos bibliográficos ni la posibilidad de consulta electrónica de datos. El tiempo máximo para contestar las preguntas fue de 30 minutos, mientras los investigadores supervisaban y verificaban el proceso. Las preguntas que tuvieron más de una respuesta seleccionada se consideraron incorrectas. Dentro de los criterios de inclusión se tuvo en cuenta que fueran estudiantes activos del pregrado de Enfermería, mayores de edad, que se encontraran en las matrículas anteriores a la tercera y superiores a la octava. De éstos, se excluyeron los que tenían estudios previos en ciencias de la salud y lo que se encontraran laborando en al área de la salud en el momento de la aplicación de la herramienta.

\section{Análisis de los datos}

Para comparar las variables Aspectos sociodemográficos y Conocimientos en TB, se distribuyeron los estudiantes en dos grupos: los que habían cursado las tres primeras matrículas y los que habían cursado desde la octava en adelante. Se asignó un cero (0) para las respuestas incorrectas y un uno (1) por cada acierto para establecer un puntaje final discriminado por estudiante y grupo de matrícula. La información se consolidó en una base de datos en Microsoft Excel $^{\circledR} 2010$ y se analizó en el programa SPSS Versión 21 en español, empleando medidas de tendencia central y de dispersión para variables continuas. Se determinó la relación entre el número de asignaturas cursadas y las matrículas con el total de conocimientos, usando el coeficiente de correlación de Spearman, ya que éste es utilizado en información que no procede de una distribución normal. Para la interpretación de la correlación, se tuvo en cuenta la clasificación propuesta por Dugarte Peña, quien afirma que Rho 0,3 $<\mathrm{r}<0,6$ es una correlación deficiente y Rho $0,6<\mathrm{r}<0,8$ es una correlación regular ${ }^{15}$. 


\section{RESULTADOS}

Cabe resaltar que éste es un estudio de alcance descriptivo, por lo que los resultados solamente permiten especificar el nivel de conocimientos sobre TB en los estudiantes participantes, es decir: se pretendió medir qué tanto sabían acerca de la enfermedad. Para la presente investigación participaron 119 estudiantes, de los cuales el $62,2 \%$ fue del género femenino y el $36,1 \%$, masculino; el 1,7\% no seleccionó ninguna opción. Las edades comprendieron desde los 18 hasta los 40 años. En cuanto al estado civil, el 88,2\% eran solteros, el 0,8\% divorciados, el 5,9\% vivían en unión libre y el 4,2\% eran casados; el 0,9\% no eligió ninguna opción. El 36,1\% había cursado las tres primeras matrículas y el $63,9 \%$ de la octava en adelante. El $84,9 \%$ de los estudiantes aseguraron que dentro de su formación académica —entre 1 y 6 asignaturas- habían recibido información sobre la TB y solamente 18 estudiantes correspondientes a las tres primeras matrículas afirmaron no haber visto asignaturas relacionadas con el tema.

\section{Conocimientos en relación a la TB}

En la variable conocimientos se identificó que los estudiantes de las últimas matrículas -o sea: de la octava en adelantedemostraron mayores conocimientos sobre TB al tener más aciertos en sus respuestas, comparado con los estudiantes de las primeras tres matrículas. Éstos evidenciaron una concentración de entre 4 y 8 aciertos, mientras que los de las últimas matrículas, entre 9 y 13 (ver Figura 1).

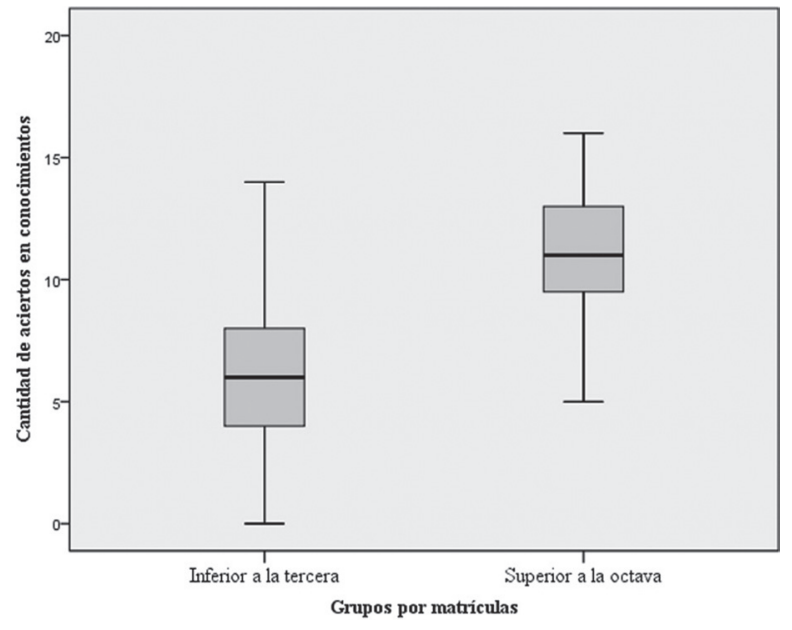

FIGURA 1. Diagrama de cajas sobre los aciertos en la variable conocimientos sobre tuberculosis de acuerdo a los dos grupos de matrículas, periodo 2013-II

De acuerdo con el anterior resultado, se encontró que hay un mejor nivel de conocimientos hacia el final de la formación, con un promedio de 11,1, mientras que en las primeras tres matrículas se obtuvo un promedio de 5,7 . Ningún estudiante respondió todas las preguntas correctamente, sólo una persona alcanzó un máximo de 16 aciertos -de la octava matrícula- y 4 estudiantes -de la primera matrícula- no tuvieron ninguna respuesta correcta (ver Tabla 1).

TABLA 1. Porcentaje de distribución de aciertos por grupos de matrículas sobre conocimientos en tuberculosis, 2013-II

\begin{tabular}{lcccc}
\hline \multicolumn{1}{c}{ Pregunta } & \multicolumn{2}{c}{$\begin{array}{c}\text { Matrículas inferiores a } \\
\text { la tercera (\%) }\end{array}$} & $\begin{array}{c}\text { Matrículas superiores a } \\
\text { la octava (\%) }\end{array}$ \\
\cline { 2 - 4 } & Correcta & Incorrecta & Correcta & Incorrecta \\
\hline ¿Cuál es la etiología de la tuberculosis? & $20(46,5)$ & $23(53,5)$ & $61(80,3)$ & $15(19,7)$ \\
¿Cuál es el agente causal de la tuberculosis? & $29(67,4)$ & $14(32,6)$ & $72(94,7)$ & $4(5,3)$ \\
¿Cuál es la principal vía de transmisión del Mycobacterium tuberculosis? & $33(76,7)$ & $10(23,3)$ & $76(100)$ & 0 \\
¿Cuál es la utilidad de vacuna BCG? & $7(16,3)$ & $36(83,7)$ & $35(46,1)$ & $41(53,9)$ \\
¿Qué significa la sigla DOTS/TAES? & $6(14,0)$ & $37(86,0)$ & $32(42,1)$ & $44(57,9)$ \\
¿Cuál es la duración general del tratamiento para un caso nuevo de tuberculosis? & $7(16,3)$ & $36(83,7)$ & $37(48,7)$ & $39(51,3)$ \\
¿Qué es un paciente sintomático respiratorio? & $8(18,6)$ & $35(81,4)$ & $41(53,9)$ & $35(46,1)$ \\
¿Cuáles son los fármacos de primera línea para un caso nuevo de tuberculosis? & $10(23,3)$ & $33(76,7)$ & $42(55,3)$ & $34(4,7)$ \\
¿Qué es la tuberculosis farmacorresistente (MDR)? & $6(14,0)$ & $37(86,0)$ & $25(32,9)$ & $51(67,1)$ \\
¿Qué es la tuberculosis extremadamente farmacorresistente (XDR)? & $3(7,0)$ & $40(93,0)$ & $22(28,9)$ & $54(71,1)$ \\
\hline
\end{tabular}

Fuente: Datos de la investigación.

El 66,4\% de los participantes comprendía la etiología de la TB y sabía correctamente que el agente causal es el Mycobacterium tuberculosis, de los cuales el 50,4\% era de últimas matrículas. El 36,6\% no dio cuenta de conocer estas nociones. Con respecto a la utilidad de la vacuna $\mathrm{BCG}$ en recién nacidos, sólo el $35,3 \%$ de los 
participantes contestó acertadamente que es útil para la prevención de la TB meníngea en menores de 1 año, frente a un $34,5 \%$ que contestó incorrectamente. El $30,3 \%$ afirmó no saber.

Acerca de la pregunta sobre la definición clínica del paciente sintomático respiratorio, el $41,2 \%$ del total de los estudiantes seleccionaron acertadamente que es la persona que presenta tos con o sin expectoración por más de 15 días, de los cuales 8 eran de primeras matrículas y 41 de las últimas. Entretanto, el 47,1\% seleccionó respuestas incorrectas y el 11,8\% respondió que no sabía qué es un paciente sintomático.

Sobre la sigla DOTS/TAES, el 31,9\% de los estudiantes conocía su significado: 6 de las primeras matrículas y 32 de las últimas. El 12,6\% seleccionó opciones incorrectas y el 55,5\% aseguró no saber lo que significa.

Al preguntar sobre los medicamentos que se utilizan para un paciente con TB sensible, el $43,7 \%$ del total de los participantes seleccionó acertadamente que son los fármacos isoniazida, etambutol, fifampicina, estreptomicina y pirazinamida. El 17,6\% eligió incorrectamente y el 38,7\% manifestó no saber. Los aciertos se presentaron en 10 estudiantes de las primeras matrículas y en 42 de las últimas.

Conforme a la definición de la MDR TB (Tuberculosis Multidrogorresistente) el 26,1\% de los encuestados sabía que es el caso en que las cepas Mycobacterium tuberculosis presentan resistencia a los fármacos isoniacida y rifampicina ${ }^{17}$. En relación con la definición de XDR TB (Tuberculosis Extremadamente Drogorresistente), se encontró que el $21 \%$ de los participantes acertó en que es el tipo de TB que presenta resistencia a la isoniacida y la rifampicina, las fluoruroquinolonas y la amikacina ${ }^{17}$. El $63 \%$ señaló no saber (ver Tabla 1).

Utilizando el coeficiente de correlación de Spearman entre los grupos de matrículas y el total de aciertos en conocimientos, se encontró un rho de 0,68 . En cuanto a los grupos de matrículas en relación con el número de materias cursadas, se halló un rho de 0,63. En la relación entre el número de asignaturas cursadas con el total de aciertos en conocimientos, se obtuvo un rho de 0,51 .

De este resultado se concluye que aunque se evidenciaron vacíos en los conocimientos de los estudiantes de enfermería, existe una relación directamente proporcional entre las variables grupo de matrículas y total de aciertos en los conocimientos. En otras palabras: a medida que avanzan los estudiantes en su formación como enfermeros, los conocimientos que adquieren aumentan, a pesar de que las asociaciones sean deficientes "matrícula con conocimientos y asignaturas con conocimientos" y regulares "asignaturas con conocimientos".

\section{DISCUSIÓN}

Una base teórica apropiada en TB permite a los estudiantes detectar oportunamente a los sintomáticos respiratorios, de manera que puedan adquirir las habilidades de diagnóstico precisas para brindar un adecuado tratamiento. De igual forma, tendrán que apoyar la creación de mecanismos para prevenir la infección por Mycobacterium tuberculosis y evitar la aparición de resistencias a los medicamentos antituberculosos, disminuyendo así la incidencia, la prevalencia y la mortalidad por la enfermedad ${ }^{12}$.

De otro lado, la OMS recomienda a los países incluir la aplicación de la vacuna BCG en los programas de inmunización. En Colombia, por ser zona de mediano riesgo de transmisión de $M$. tuberculosis, los niños se encuentran expuestos a una mayor probabilidad de contraer la TB miliar o la meníngea, por eso la vacuna se administra a los recién nacidos en única dosis, de acuerdo a los lineamientos del Programa Ampliado de Inmunización y la Guía de Atención de la Tuberculosis Pulmonar y Extrapulmonar (PAI) $)^{4,18}$.

No obstante, esta investigación identificó que los estudiantes participantes revelaron bajos aciertos en los resultados, es decir: falencias en los conocimientos sobre la TB, específicamente en la utilidad de la vacuna BCG, la definición de paciente sintomático respiratorio, la estrategia DOTS/TAES, la terapia farmacológica y la definición de MDR TB y XDR TB. A pesar de estos resultados, los investigadores del presente estudio hallaron que los estudiantes de enfermería aumentan el nivel de conocimientos relacionados con la TB en el transcurso de la formación académica, lo cual se corroboró con el coeficiente de correlación de Spearman.

Estudios realizados en Brasil ${ }^{11}$, Irán ${ }^{9}$ y Turquía $^{10}$ arrojaron resultados similares a los del presente estudio, en los que se evidenció que la mayoría de los encuestados desconocía la utilidad de la vacuna BCG y su esquema de aplicación de acuerdo a cada país. Aun al haber hallado desconocimiento en la temática, se identificó que el grupo de las últimas matrículas 
obtuvo una diferencia del 29,8\% (ver Tabla 1) superior con respecto al grupo de las primeras, lo que de todas maneras es preocupante, ya que los profesionales de enfermería son los encargados del manejo y control de la vacuna $\mathrm{BCG}$.

En Italia, Laurenti y cols. en su estudio midieron los conocimientos sobre TB en estudiantes de postgrado de especialidades médicas, encontrando que el $90 \%$ sólo conocía el nombre de la vacuna19. Si bien eran estudiantes de un nivel de formación superior al de pregrado, desconocían los elementos básicos sobre la vacuna. Sin embargo, este estudio no indagó sobre la utilidad de la BCG.

En los trabajadores de la salud que manejan los programas de control de la TB en la ciudad de Bogotá, Cruz y et al., encontraron semejanzas en el desconocimiento de la utilidad de la vacuna BCG, en el que sólo el 60,5\% identificó la importancia que reviste en la prevención de la TB. Es inquietante que estos profesionales lideren los programas de control, ya que los roles deben ser asumidos a partir de conocimientos proporcionales a sus responsabilidades ${ }^{8}$.

Es necesario que los estudiantes y profesionales de enfermería posean la información fundamental y se mantengan actualizados sobre la vacuna como una herramienta para el control de la TB, puesto que el profesional de enfermería es el que lidera en varios países los programas de vacunación y aplica los cuidados en indicaciones, administración, conservación del biológico, efectos secundarios, contraindicaciones y en la utilidad de la vacuna ${ }^{11}$.

En 1993, la OMS declaró la TB como una emergencia mundial en salud pública, por ello se creó la estrategia DOTS/TAES en 1995 con la consolidación de sus cinco elementos, para posteriormente implementarla en el continente americano en 1996, a partir de lo cual se obtuvieron grandes avances de hasta el $80 \%$ de la cobertura. No obstante, en esta investigación se halló que menos de la tercera parte de los estudiantes encuestados sabía el significado de la estrategia DOTS/TAES, a pesar de que la literatura científica la documenta como la estrategia más efectiva en el control de la TB a nivel mundial ${ }^{4}$.

Así mismo, en un estudio descriptivo en Cuba (20112012) por Abreu y et al., se encontró que el $46,6 \%$ de los estudiantes de medicina encuestados desconocían la importancia de la estrategia DOTS/TAES, pese a haber participado en seminarios y conferencias sobre TB. Sin embargo, con respecto a los estudiantes del presente estudio, se evidenció inconsistencias en el nivel de nociones que debían dominar de la estrategia, pues 38 apenas identificaban la sigla. Además se encontró que tuvieron menores resultados en comparación con el estudio de Cuba, con una diferencia del 14,7\% menor en los conocimientos referentes a la estrategia. El resultado en Turquía abre aún más la brecha al poseer un porcentaje menor en relación con la población diana de esta investigación, pero coinciden en que los estudiantes de últimos semestres demostraron mayores conocimientos ${ }^{12}$. En cambio, Zhao et al. (2011-2012) concluyeron que solamente el 14,5\% de los participantes de su estudio (1 486 estudiantes) era plenamente consciente de la estrategia DOTS/ TAES y menos del $10 \%$ estaba familiarizado con sus cinco elementos ${ }^{20}$. En relación con los estudiantes de la presente investigación, se encontró que el grupo de las primeras matrículas tenía el mismo porcentaje de aciertos que los del estudio de China, lo que significa que la totalidad de participantes de este estudio tuvieron mayores aciertos.

En la India, Kutare y et al, realizaron un estudio con 207 internos de tres escuelas de medicina, cuyo herramienta mostró que el $47,4 \%$ de los encuestados no adquirió la información sobre el DOTS/TAES durante su formación. Aun así, en comparación con el presente estudio, los participantes de Kutare y et al., tuvieron más aciertos al tener una diferencia a favor del $20,7 \%{ }^{21}$.

En este sentido, cuando el equipo de salud presenta insuficientes conocimientos en el tratamiento de la TB, afecta el desarrollo del programa de control planteado en cada país. Se destaca que la estrategia DOTS/TAES es considerada la más costo-efectiva y con mayores beneficios en el control de la TB. Por lo tanto, su desconocimiento puede menoscabar considerablemente el éxito de la ejecución, con el consecuente incremento en las tasas de abandono del tratamiento, la aparición de farmacorresistencias y el incremento en las cifras de mortalidad $^{4,22}$.

La estrategia DOTS-TAES trae efectos positivos adicionales a la curación y disminución de la resistencia a los medicamentos: un vínculo más estrecho entre enfermeros y sujetos de cuidado; identificación de necesidades de la población enferma; canalización del acceso a programas sociales; acciones educativas en salud y favorecimiento de una vida digna ${ }^{23}$. Por esta razón, la estrategia debe prevalecer en la formación académica de las universidades y consolidarse como lo estipula la OPS: utilizar estrategias pedagógicas, como la consulta 
a expertos, espacios de discusión y situaciones prácticas, ya que motivan al pensamiento crítico y potencian el compromiso para la atención en salud ${ }^{4}$.

En lo que respecta a la terapia farmacológica, se encontró que menos de la mitad de los estudiantes de enfermería demostró conocimientos sobre los fármacos utilizados en la curación y la duración de un caso nuevo de TB pulmonar activa. Se evidenció que tan sólo un $21,8 \%$ del total de los encuestados identificaron el esquema del tratamiento y su duración. Entre las dificultades para el control y erradicación de la TB, se encuentran las cepas resistentes a los antibióticos. Una de sus casusas es la resistencia de tipo iatrogénica, es decir: durante la formación de los profesionales de la salud no hay claridad en qué medicamentos utilizar, cuánto es la dosificación exacta, cuál es la vía de administración o cuál es su conservación ${ }^{24}$. En estudios realizados a estudiantes de ciencias de la salud que indagaron por el tratamiento de la TB, Akin y et al. identificaron que solamente el $29,1 \%$ de sus estudiantes de enfermería conocía la duración del tratamiento, el $24.6 \%$, la dosis de cada fármaco y el 27,3\%, el control de los efectos secundarios ${ }^{10}$. Resultados similares arrojó la investigación de Zhao en estudiantes de medicina con respecto a la duración ${ }^{20}$, pero tuvieron porcentajes más altos -con una diferencia del 39\%- los hallados por Mehta en su estudio ${ }^{25}$. No obstante, no hubo diferencias significativas entre los estudiantes que se encontraban o no en prácticas clínicas, como sucedió en los estudiantes del presente estudio, cuyo grupo de últimas matrículas obtuvo mejores resultados, ya que éste había tenido experiencias prácticas en rotaciones de área hospitalaria y comunitaria, mientras que el grupo de las primeras matrículas no había iniciado su experiencia práctica ${ }^{5,21,22}$.

En el estudio de Cruz y et al, se encontró que el 64\% de los trabajadores de la salud conocía los fármacos de primera línea en el esquema de tratamiento ${ }^{8}$, mientras que los estudios realizados en Perú y Turquía revelaron que casi todos los enfermeros conocían los fármacos de primera línea y la duración del tratamiento, lo que conlleva a que factores como la edad, la experiencia y mejores niveles de estudio influyan en poner en práctica un mejor control de la $\mathrm{TB}^{26,27}$.

La MDR TB y XDR TB se han convertido en una amenaza para el control de la TB, inclusive para el cumplimiento del sexto objetivo de los ODM para el 2015, ya que se ha reportado más de un tipo de resistencias a los fármacos antituberculosos a nivel mundial y un aumento anual del número de casos, de acuerdo al informe mundial ${ }^{1}$. En el 2012 se presentaron en Las Américas 28.625 casos de MDR TB y en Colombia se reportaron entre el 3\% y el 6\% de los casos de incidencia en los respectivos sistemas de vigilancia epidemiológica ${ }^{1,28}$.

En este aspecto, se evidenció que los estudiantes participantes del presente estudio tuvieron, en comparación con las demás preguntas, los más bajos porcentajes de conocimientos en esta temática, caso que no difiere demasiado de los estudios CAP. Por ejemplo: Abreu y et al, encontraron que la mitad de los estudiantes de medicina afirmaron que la MDR TB es aquella que presenta resistencia en todos los fármacos antituberculosos de primera línea, señalamiento a todas luces erróneo ${ }^{12}$. La situación en los trabajadores de la salud no se diferencia mucho del caso anterior. Kiefer encontró que menos de la mitad de los trabajadores conocía que la TB resistente y su propagación eran consecuencias de un tratamiento inadecuado o incompleto. Basu, por su parte, halló que apenas la cuarta parte de los médicos tenía conocimiento sobre la MDR TB ${ }^{29,30}$.

Menos de la mitad de los estudiantes de la presente investigación conocía la definición clínica del paciente sintomático respiratorio, lo cual contrasta con el estudio de los estudiantes de medicina en Pereira, en el que el $90 \%$ los estudiantes acertaron en que la tos es uno de los síntomas 7 . Por su parte, Zhao encontró que sólo el $24 \%$ de los estudiantes sabía que la tos con esputo teñido de sangre (hemoptisis) es el síntoma clásico de la TB, aunque cabe aclarar que la tos, independiente de otros signos y síntomas, ya es sospecha de TB. Zhao observó también que los resultados con respecto a esta pregunta tienden a mejorar levemente en cada año de formación, similar a lo hallado en la presente investigación ${ }^{20}$.

Entender la definición clínica del sintomático respiratorio le permite al profesional de enfermería identificar oportunamente a las personas enfermas de TB, de manera que pueda llevar a cabo las respectivas pruebas diagnósticas e iniciar prontamente la terapia farmacológica correspondiente. Es por esta razón que el profesional de la salud está en la obligación de reconocer a quien presente tos por más de quince días con o sin esputo -independientemente de que esté acompañada de otros síntomas como inapetencia, pérdida de peso o sudoración nocturna-como paciente con sospecha de $\mathrm{TB}^{4,22}$. 


\section{SAabus 담}

Olarewaju en su investigación identificó que los estudiantes reconocieron la necesidad de fortalecer los conocimientos de esta enfermedad, para lo cual es menester que la problemática se aborde desde las universidades y los hospitales universitarios, aunque la mayoría se sienta seguro de que los conocimientos adquiridos son suficientes para suplir las necesidades en la atención ${ }^{31}$.

En las investigaciones cualitativas de Muñoz, Woith, Mathew y Wannhedenen, los trabajadores de la salud también reconocieron sus vacíos en conocimientos y la falta de experiencia en el abordaje del paciente con TB. Sostienen que en las universidades la educación se enfatiza más en los procesos clínicos que en los asuntos de salud pública, proponiendo como solución un cambio en el paradigma de la formación, y que así se asocie más a la temática de salud pública y colectiva que a la clínica ${ }^{32-35}$.

\section{CONSIDERACIONES FINALES}

Los bajos porcentajes de conocimientos encontrados en los estudiantes de enfermería pueden tener implicaciones como: vulnerabilidad al contagio por la exposición al agente causal en las prácticas formativas; fallas en la implementación del programa para el control de la tuberculosis; posible aumento en las tasas de incidencia y mortalidad, tanto en los estudiantes como en la población; disminución en el grado de responsabilidad de los estudiantes en el abordaje de esta enfermedad; y favorecimiento de la aparición de TB farmacorresistente.

Aunque procuran mejorar en el transcurso de la formación, se evidenciaron deficiencias en el nivel de conocimientos en el presente estudio, por lo cual se sugiere realizar estudios de intervención que permitan una mejor apropiación en la temática. En consecuencia, se recomienda articular constantemente la formación académica con la problemática de TB en el país, entendiendo el proceso salud/enfermedad como un fenómeno biológico, patológico, social, económico, político, ambiental y cultural, desde las comunidades y las instituciones de salud.

\section{AGRADECIMIENTOS}

A la División de Investigación de la Universidad Nacional de Colombia, Sede de Bogotá, por el apoyo financiero a través del programa nacional de semilleros de investigación, creación e innovación de la Universidad Nacional de Colombia 2013-2015, bajo el código 18146.

Partiendo que este estudio es un proyecto derivado de semilleros de investigación, la Facultad de Enfermería de la Universidad Nacional de Colombia ha definido que el docente tutor debe garantizar los aspectos éticos, según la Resolución No. 077 del 2006 del Consejo de Facultad de Enfermería.

De acuerdo al Artículo 11 de la Resolución 008430 el Ministerio de Salud, esta investigación se clasifica en la categoría Investigación sin riesgo, una vez que no se efectuaron intervenciones o modificaciones intencionadas de los aspectos biológicos, psicológicos o sociales de los estudiantes participantes. Se elaboró un consentimiento informado con el objeto de dar la información pertinente a los participantes acerca del proyecto de investigación, sus contenidos y el uso de los datos recolectados, garantizando los cuatro principios de la bioética: no maleficencia, beneficencia, justicia y autonomía. Cada estudiante firmó de manera voluntaria su participación 16.

\section{CONFLICTO DE INTERÉS}

Los autores del presente manuscrito declaran no tener algún conflicto de interés.

\section{REFERENCIAS}

1. Organización Mundial de la Salud (OMS). The burden of disease caused by TB. In: Global Tuberculosis Report 2014. Francia: minimum graphics; 2014: 7-32.

2. Organización Panamericana de la Salud (OPS). La carga regional de tuberculosis. En: La Tuberculosis en la Región de las Américas, informe regional 2012: epidemiología, control financiero. Washington, DC.; 2013.

3. Instituto Nacional de Salud. Boletín epidemiológico semanal, semana epidemiológica número 51 de 2013. Bogotá; 2013: 1-25.

4. Organización Panamericana de la Salud (OPS). Enseñanza de la tuberculosis en las facultades de salud: informe de una consulta de expertos. Cartagena, Colombia; 2004: 1-39.

5. Teixeira EG, Menzies D, Cunha AJ, Luiz RR, Ruffino-netto A, Scartozzoni MS, et al. Knowledge and practices of medical students to prevent Tuberculosis transmission in Rio de Janeiro, Brazil. Rev Panam Salud Publica. 2008; 24(4): 265-270.

6. Organización Mundial de la Salud (OMS). A guide to developing knowledge, attitude and practice 
surveys. Suiza; 2008: 1-68.

7. Castañeda Hernández DM, Mondragón Cardona BÁ, Campo Betancourt CF, Tobón García D, Alzate Carvajal V, Jiménez Canizales CE, et al. Impacto de una actividad formativa en los conocimientos, actitudes y percepciones sobre Tuberculosis de estudiantes de medicina de una Universidad de Risaralda, Colombia. Gac méd Caracas. 2012; 120(1): 40-47.

8. Cruz Martínez ÓA, Flórez Suancha ÉL, Muñoz Sánchez AI. Conocimientos sobre Tuberculosis en trabajadores de la salud en una localidad de Bogotá D.C. Rev Av en Enfermería. 2011; 29(1): 143-151.

9. Behnaz F, Mohammadzade G, Mousavi-eRoknabadi RS, Mohammadzadeh M. Assessment of knowledge, attitudes and practices regarding tuberculosis among final year students in Yazd, Central Iran. J Epidemiol Glob Health. 2014; 4(2): 81-85.

10. Akin S, Gorak G, Unsar S, Mollaoglu M, Ozdilli K, Durna Z. Knowledge of and attitudes toward tuberculosis of Turkish nursing and midwifery students. Nurse Educ Today. 2011; 31(8): 774-779.

11. Mussi TV, Traldi MC, Talarico JN. Knowledge as a factor in vulnerability to tuberculosis among nursing students and professionals. Rev Esc Enferm USP. 2012; 46(3): 696-703.

12. Abreu Suárez CG, González Valdés JA, Muñoz Peña R, Solar Salaverri LA, Marchena Béquer JJ. La preparación del estudiante de Medicina para la eliminación de la tuberculosis. Educ Médica Super. 2013; 27(1): 38-45.

13. Ministerio de la Protección Social. Plan Estratégico Colombia Libre de Tuberculosis 2006-2015: Para la Expansión y Fortalecimiento de la Estrategia Alto a la Tuberculosis. 3Ed. Bogotá: Gráficas Ducal; 2007.

14. Republica de Colombia. Ministerio de salud. Resolución Número 412 del 25 de febrero de 2000, por la cual se establecen las actividades, procedimientos e intervenciones de demanda inducida y obligatorio cumplimiento y se adoptan las normas técnicas y guías de atención para el desarrollo de las acciones de protección específica y detección temprana y la atención de enfermedades de interés en salud pública. Bogotá: Ministerios de Salud; 2000: 1-6.

15.Dugarte Peña E. Estadística y probabilidad. 1Ed. Bucaramanga: Pontificia Universidad Bolivariana; 2013.

16. Republica de Colombia. Ministerio de salud. Resolución 8430 del 4 de octubre de 1993, por la cual se establecen las normas científicas, técnicas y administrativas para la investigación en salud.
Ministerio de salud; 1993: 1-12.

17. Instituto Nacional de Salud, Organización Panamericana de la Salud (OPS). Lineamientos manejo de Tuberculosis Farmacorresistente. Bogotá; 2013.

18. Ministerio de Salud y Proteccion Social. Lineamientos para la gestión y administración del programa ampliado de inmunizaciones - PAI 2013. 2012.

19. Laurenti P, Federico B, Raponi M, Furia G, Ricciardi W, Damiani G. Knowledge, experiences, and attitudes of medical students in Rome about Tuberculosis. Med Sci Monit. 2013; 19: 865-874.

20. Zhao Y, Ehiri J, Li D, Luo X, Li Y. A survey of Tuberculosis knowledge among medical students in Southwest China: is the information reaching the target? BMJ Open. 2013; 3(9):1-10.

21. Kutare A, Rosario M, Goudb N. A Study on Knowledge of Tuberculosis, DOTS and MDR-TB among Interns of Medical Colleges in Bangalore. Int J Heal Sci Res. 2012; 2(3): 33-39.

22. Alarcón E. Guía de Enfermería para la implementación y expansión de la estrategía DOSTTAES. Paris, Francia; 2004: 70.

23. Muñoz Sánchez AI, Bertolozzi MR. Más allá del DOTS (Directly Observed Treatment ShortCourse) en el control de la tuberculosis: medio que promueve la comunicación y la identificación de las necesidades. Rev Latino-am Enferm. 2009; 17(5): 689-694.

24. Vargas R, Bayona M, Ante LA. Tuberculosis una enfermedad del ayer del hoy y del futuro. Med Ac Col. 2013; 35(3): 227-236.

25. Mehta D, Singh M, Bassi R, Mehta C. To study the knowledge about Tuberculosis management and national tuberculosis program among medical students and aspiring doctors in a high tubercular endemic country. Ann Trop Med Public Heal. 2012; 5(3): 206-208.

26. Yükseltürk N, Dinç L. Knowledge about antituberculosis treatment among nurses at tuberculosis clinics. Int J Nurs Pract. 2013; 19(1): 47-53.

27. Minnery M, Contreras C, Pérez R, Solórzano N, Tintaya K, Jimenez J, et al. A cross sectional study of knowledge and attitudes towards tuberculosis amongst front-line tuberculosis personnel in high burden areas of Lima, Peru. PLoS One. 2013; 8(9): e75698.

28. Mukherjee A, Lodha R, Kabra SK. Recent advances in diagnosis of Tuberculosis. Pediatr Infect Dis. 2012; 4(2): 45-50.

29. Kiefer E, Shao T, Carasquillo O, Nabeta P, Seas C. Knowledge and attitudes of tuberculosis 
management in San Juan de Lurigancho district of Lima , Peru. J Infect Dev Ctries. 2009; 3(10): 783-788.

30. Basu M, Sinha D, Das P, Roy B, Biswas S, Chattopadhyay S. Knowledge and practice regarding pulmonary tuberculosis among private practitioners. Indian J community Heal. 2013; 25(04): 403-412.

31. Olakunle OS, Oladimeji O, Olalekan AW, Olugbenga-Bello A, Akinleye C, Oluwatoyin OA. Knowledge of tuberculosis management using directly observed treatment short course therapy among final year medical students in South Western Nigeria. Pan Afr Med J. 2014; 18(32): 1-12.

32. Woith W, Volchenkov G, Larson J. Barriers and motivators affecting tuberculosis infection control practices of Russian health care workers. Int $\mathrm{J}$
Tuberc Lung Dis. 2012; 16(8): 1092-1096.

33. Wannheden C, Westling K, Savage C, Sandahl C, Ellenius J. HIV and tuberculosis coinfection: a qualitative study of treatment challenges faced by care providers. Int J Tuberc Lung Dis. 2013; 17(8): 1029-1035.

34. Muñoz Sánchez AI, Cruz Martínez ÓA, Rubiano Mesa YL. Trabajadores de la salud y sus significados en torno a la adherencia al tratamiento de la tuberculosis. Enfermería Glob. 2013; 31: 86-108.

35. Mathew TA, Shields AL, Imasheva A, Shin SS, Mishustin SP, Peremitin GG, et al. Knowledge, attitudes, and practices of physicians in Tomsk Oblast tuberculosis services regarding alcohol use among tuberculosis patients in Tomsk, Russia. Cult Med Psychiatry. 2009; 33(4): 523-537. 